\title{
Acute childhood encephalopathy with prolonged fever, pleocytosis and elevated inflammatory cytokines in the cerebrospinal fluid and transient splenial lesion
}

\author{
Rena Okada $^{1}$; Yuri Sakaguchi ${ }^{1}$; Takeshi Matsushige ${ }^{2}$; Isamu Kamimaki ${ }^{3}$; Toshiki Takenouchi ${ }^{1}$; \\ Takao Takahashi ${ }^{1}$ \\ ${ }^{1}$ Department of Pediatrics, Keio University School of Medicine \\ ${ }^{2}$ Department of Pediatrics, Yamaguchi University Graduate School of Medicine \\ ${ }^{3}$ Department of Pediatrics, National Hospital Organization, Saitama National Hospital \\ Corresponding author: Yuri Sakaguchi; Email: cardinal@keio.jp. \\ (6) https://doi.org/10.17724/jicna.2019.166
}

Received: 15 May 2019

Accepted: 21 September 2019

\begin{abstract}
Background: Acute encephalopathy during childhood represents a highly heterogeneous group of infectious and noninfectious pathologies. According to a recent nationwide survey on acute childhood encephalopathy in Japan, the combination of clinical and radiographic features left approximately half of the affected children unclassified, mainly because of the lack of disease-specific biomarkers.
\end{abstract}

Case: Herein, we document a school-aged boy who manifested with acute encephalopathy that was characterized by a prolonged fever, altered mental status, urinary retention, and intention tremor lasting for more than a month. Accompanying features included syndrome of inappropriate secretion of antidiuretic hormone, pleocytosis with elevated interleukin-6 and interferon-gamma levels in the cerebrospinal fluid, and a transient splenial lesion on neuroimaging. No pathogens were identified, and C-reactive protein was negative throughout his clinical course. This constellation of clinical features was not compatible with any of the existing entities of acute pediatric encephalopathy.

Discussion: Our retrospective literature review identified two additional school-aged male patients who exhibited highly similar clinical courses. The prolonged altered mental status with pleocytosis in the cerebrospinal fluid and a transient splenial lesion in the absence of serum inflammatory markers suggest a primary central nervous system pathology.

Conclusion: This combination of features defines this presumably new group of acute childhood febrile encephalopathy with prolonged fever and ataxia in school-aged boys.

Keywords: ataxia; cytokine; febrile encephalopathy; hyponatremia; pleocytosis; splenium of the corpus callosum; urinary retention.

(c) Okada R. et al.; licensee JICNA.

\section{Introduction}

Acute childhood encephalopathy is predominantly seen in East Asian countries and comprises a heterogeneous group of infectious and non-infectious disorders [1]. However, in most cases, the exact molecular mechanisms remain unknown because of the lack of disease-specific biomarkers. Over the past few decades, attempts have been made to classify acute childhood encephalopathy based primarily on clinical features $[1,2,3,4,5]$. As a result of a recent series of nationwide surveys conducted by Mizuguchi, et al., 2007 - 2010 [6], our understanding of this highly heterogeneous group of patients has progressed significantly to the point where up to $50 \%$ of affected children can be classified based on a combination of clinical and radiographic features, even in the absence of specific biomarkers. However, approximately half of all affected children remain unclassifiable. In this report, we document a boy who manifested acute encephalopathy with prolonged fever, an altered mental status, and a tremor lasting for more than one month. This highly unusual clinical course did not correspond to any of the existing groups of childhood encephalopathy. 


\section{Case Report}

A previously healthy nine-year-old boy presented with a subacute onset of fever of $39{ }^{\circ} \mathrm{C}$, frontal throbbing headache, and vomiting. He was seen at a local hospital, where his physical examination and routine laboratory tests were uninformative. He was diagnosed as having an unknown viral infection. On day six, he returned to the local hospital because of a persistent high fever and intermittent headaches, and was barely able to walk by himself. He was also drowsy and slow to respond.

On day 13, he was transferred to a tertiary care centre for further medical evaluation. On admission, his vital signs were body temperature of $39.5^{\circ} \mathrm{C}$, heart rate of 109 beats per minute, respiratory rate of 20 breaths per minute, and blood pressure of 104/64 mmHg. His physical examination was unremarkable with no signs of meningism, but he complained of chills and posterior neck pain, and had marked ataxia with intention tremor. He was able to stand by himself for only a few seconds with a tendency to fall, and was drowsy and confused as to the time and date. His speech was incoherent, for example: "Bring me my sushi, I am going to eat them at 8 o'clock". He also had urinary retention and incontinence.

The cerebrospinal fluid [CSF] examination on day 8 revealed a cell count of $355 / \mu \mathrm{L}$ with lymphocyte dominance. The cytokine profile in his CSF was abnormal: his IL-6 level was elevated to $157.4 \mathrm{pg} / \mathrm{mL}$ [reference range: < $6.2 \mathrm{pg} / \mathrm{mL}$ ] and his IFN- $\gamma \gamma$ level was elevated to 266.3 $\mathrm{pg} / \mathrm{mL}$ [reference range: $<7.1 \mathrm{pg} / \mathrm{mL}$ ]. He developed hyponatremia with a level of $124 \mathrm{mEq} / \mathrm{L}$ accompanied by an increased level of antidiuretic hormone, consistent with a diagnosis of syndrome of inappropriate secretion of antidiuretic hormone [SIADH]. No pathogens, bacterium or virus, including influenza, were identified in his peripheral blood, CSF, and pharynx. He remained negative for C-reactive protein throughout his clinical course. Magnetic resonance imaging [MRI] of the brain was performed on day 8, revealing a hyperintensity within the splenium of the corpus callosum on the diffusion-weighted imaging sequence [TE $=55 \mathrm{~ms}$ and $\mathrm{TR}=2828.6 \mathrm{~ms}$ ]. An electroencephalogram on day 9 showed a posterior predominant slowing in the delta frequency range. The patient was treated with intravenous methylprednisolone at $30 \mathrm{mg} / \mathrm{kg} /$ day on days 9-11 and 15-17 with no resolution of his fever or his mental status. He was further treated with intravenous immunoglobulin at $400 \mathrm{mg} / \mathrm{kg} /$ day on days $22-26$, which was also ineffective.

The patient's urinary retention recovered by day 25 but he remained febrile for a total of 26 days during the course of the disease, defervescing by day 30 . His intention tremor was spontaneously resolved by day 40 . Seven months after his discharge he had not had any episodes of high fever and had no obvious residual neurological deficits. He attended a regular school class.

\section{Discussion}

We report on a nine-year-old boy who presented with subacute onset of febrile encephalopathy, characterized by a prolonged high fever with altered mental status and ataxia lasting for more than one month. The condition was accompanied by pleocytosis and an elevation of inflammatory cytokines in the CSF, i.e., IL- 6 and IFN- $\gamma$, and a transient splenial lesion on neuroimaging. The patient recovered after receiving various immune therapies, with no resultant neurological deficits. A strikingly prolonged course of febrile encephalopathy is not included in the recently proposed classification of acute childhood encephalopathy, according to the current expert consensus guidelines [7].

Through our local collaboration network and extensive literature search, we identified two additional patients who exhibited similar clinical courses [? 8]. Our review of the three patients showed that they shared the following characteristics [see Table 1]: they were all school-aged boys who had a prolonged fever, altered mental status lasting for more than three weeks, ataxia lasting for more than one month, SIADH, pleocytosis in the CSF, negative blood cultures and C-reactive protein in peripheral blood, reversible splenial lesions on neuroimaging, and no residual neurological deficits at a remote follow-up. Two of the three patients [patients 1 and 2] had urinary retention and abnormal cytokine profiling in the CSF. [This analysis was not performed on patient 3.] The constellation of these clinical, laboratory and radiographic features clearly distinguishes these three patients from those in any of the existing entities of acute pediatric encephalopathy and thus may represent a new group of acute childhood febrile encephalopathy. Given the regional presence of this condition, a genetic predisposition or a prevalent local infectious trigger may have a role in the pathogenesis of this condition.

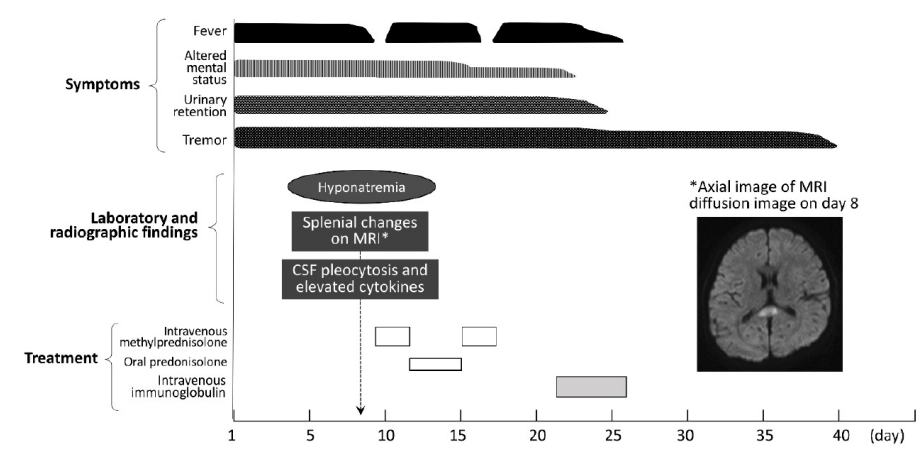

Figure 1 Schematic summary of clinical course in patient 1.

The horizontal axis represents the number of days after the onset of symptoms. Each symptom and treatment is presented as a horizontal bar. Note the prolonged fever, consciousness disturbance, urinary retention, and tremor. MRI: magnetic resonance imaging.

The presence of an altered mental status with pleocytosis in the CSF and a transient splenial lesion in the absence of serum inflammatory markers does not prove, but does 
Table 1 Summary of clinical, laboratory and neuroradiographic features in the three patients.

\begin{tabular}{|c|c|c|c|c|}
\hline Patient \# & & 1 & 2 & 3 \\
\hline References & & The present report & Takashi et al. [9] & Tsuruta et al. [8] \\
\hline Age/Gender & & 9 , male & 14 , male & 14, male \\
\hline \multirow[t]{9}{*}{ Clnical features } & Prolonged fever & Present & Present & Present \\
\hline & (duration) & $(\approx 26$ days $)$ & $(\approx 38$ days $)$ & $(\approx 30$ days $)$ \\
\hline & Altered mental status & Present & Present & Present \\
\hline & (duration) & $(\approx 22$ days $)$ & $(\approx 16$ days $)$ & $(\approx 28$ days $)$ \\
\hline & Tremor & Present & Present & Present \\
\hline & (duration) & $(\approx 40$ days $)$ & $(\approx 40-50$ days $)$ & $(\approx 50$ days $)$ \\
\hline & Urinary retention & Present & Present & Absent \\
\hline & (duration) & $(\approx 25$ days $)$ & $(\approx 27$ days $)$ & \\
\hline & outcome & No sequelae & No sequelae & No sequelae \\
\hline \multirow[t]{3}{*}{ Blood } & Hyponatremia (nadir time) & Present & Present & Present \\
\hline & & $124 \mathrm{mEq} / \mathrm{L}$ on day 6 & $120 \mathrm{mEq} / \mathrm{L}$ on day 9 & $132 \mathrm{mEq} / \mathrm{L}$ on day 7 \\
\hline & C-reactive protein & Negative & Negative & Negative \\
\hline \multirow{6}{*}{$\begin{array}{l}\text { Cerebrospinal Fluid } \\
\text { (CSF) }\end{array}$} & Cell count (number, day) & Elevated & Elevated & Elevated \\
\hline & & $355 / \mu \mathrm{L}$ on day 8 & $354 / \mu \mathrm{L}$ on day 6 & $111 \mu \mathrm{L}$ on day 7 \\
\hline & IL-6 $($ ref $<6.2 \mathrm{pg} / \mathrm{mL})$ & Elevated & Elevated & Not measured \\
\hline & (amount, day) & $157.4 \mathrm{pg} / \mathrm{mL}$ on day 8 & $258.5 \mathrm{pg} / \mathrm{mL}$ on day 6 & \\
\hline & IFN $-\gamma$ ref $<2.8 \mathrm{pg} / \mathrm{mL})$ & Elevated & Elevated & Not measured \\
\hline & & $266.3 \mathrm{pg} / \mathrm{mL}$ on day 8 & $1,805.2 \mathrm{pg} / \mathrm{mL}$ on day 6 & \\
\hline \multirow[t]{2}{*}{ Neuroimaging } & Changes in splenium of corpus callosum & Present & Present & Present \\
\hline & (day) & On day 8 & On day 8 & On day 9 \\
\hline
\end{tabular}

suggest, a primary central nervous system pathology, rather than a secondary phenomenon arising from a systemic illness. From a radiographic standpoint, transient splenial lesions have been described in association with a number of systemic conditions, such as hyponatremia, hypoxia and infections. The entire clinical picture of these three reported patients is distinct from an entity termed 'mild encephalopathy with reversible splenial lesion' [MERS] that was proposed by Takanashi et al. [5], where complete resolution of the neurological symptoms within a month of onset is the rule $[3,7]$. Moreover, in the absence of CSF inflammation, MERS is considered in most cases to be an infection-associated encephalopathy based on the presence of elevated serum inflammatory markers [10]. Nonetheless, in rare cases of MERS, elevated CSF inflammatory markers were observed [11]. Such patients may have shared pathogenesis with this presumably new entity. The plausible mechanism for splenial lesion and hyponatremia in the setting of central nervous system inflammation includes the involvement of an immune-neuroendocrine pathway [12]. The absence of a systemic inflammatory marker, i.e., C reactive protein, despite pleocytosis and elevated IL- 6 levels in the CSF, appears to be a key feature of this condition.

In conclusion, three unrelated school-aged boys presented with an unusual course of subacute febrile encephalopathy with ataxia lasting for more than one month. Accompanying features included SIADH, pleocytosis with elevated inflammatory cytokine levels in CSF, and transient splenial lesions. Further investigation on the clinical and molecular aspects in a larger cohort of patients is warranted. We propose that a combination of these clinical, laboratory and radiographic findings signifies a previously undescribed group of patients with acute childhood encephalopathy.

\section{Acknowledgments}

We thank Dr. Masaru Takayanagi from Department of Pediatrics, Sendai City Hospital, Sendai, Japan, and Dr. Makoto Tsuruta from Department of Pediatrics, Kofu Kyoritsu Hospital, Kofu, Japan for providing detailed clinical information on patients 2 and 3.

\section{Competing interests}

The authors have declared that they have no competing interest.

\section{Authors' contributions}

All the authors contributed to data collection and also critically reviewed the manuscript. The final version of the manuscript was approved by all the authors.

This is an Open Access article distributed under the terms of the Creative Commons Attribution License which permits unrestricted use, distribution, and reproduction in any medium, provided the original work is properly credited. The Creative Commons Public Domain Dedication waiver applies to the data made available in this article, unless otherwise stated.

\section{Cite this article as:}

Okada, R., Sakaguchi, Y., Matsushige, T., Kamimaki, I., Takenouchi, T., \& Takahashi, T. (2019). Acute childhood encephalopathy with prolonged fever, pleocytosis and elevated inflammatory cytokines in the cerebrospinal fluid and transient splenial lesion. Journal of the International Child Neurology Association, 1(1). https://doi.org/10.17724/jicna.2019.166 


\section{References}

[1] H Yamanouchi YA M Shiomi, Mizuguchi M. Current topics of acute encephalitis and encephalopathy in Japanese children. PNo To Hattatsu. 2012;41(2):1246. PublMed.

[2] Mizuguchi M. Acute necrotizing encephalopathy of childhood: a novel form of acute encephalopathy prevalent in Japan and Taiwan. Brain and Development. 1997 Mar;19(2):81-92. Publ(Med.

[3] ichi Takanashi J. Two newly proposed infectious encephalitis/encephalopathy syndromes. Brain and Development. 2009 Aug;31(7):521-528. PublMed.

[4] Sakuma H, Awaya Y, Shiomi M, Yamanouchi H, Takahashi Y, Saito Y, et al. Acute encephalitis with refractory, repetitive partial seizures (AERRPS): a peculiar form of childhood encephalitis. Acta Neurologica Scandinavica. 2010 Apr;121(4):251-256. Publ(Med.

[5] Nukui M, Kawawaki H, Inoue T, Kuki I, Okazaki S, Amo K, et al. Clinical characteristics of acute encephalopathy with acute brain swelling: A peculiar type of acute encephalopathy. Brain and Development. 2018 Oct;40(9):792-798. Publ(Med.

[6] M Mizuguchi M AOMKYS A Oka, Takanashi J. Nationwide Survey of Acute Childhood Encephalopathy: research on rare and intractable diseases (in Japanese). Ministry of Health, Labour and Welfare. 2012;

[7] The Japanese Society of Child Neurology e supervised Committee on Evaluation Guidelines for Pediatric Acute Encephalopathy. Evidence-based Practice Guidelines for the Treatment of Pediatric Acute Encephalopathy 2016 (in Japanese). Shindan to Chiryosha. 2016;p. 115-9.

[8] Tsuruta M UJNK Mori H. An atypical course of mild encephalopathy with reversible splenial lesion accompanied by prolonged fever and involuntary movement (in Japanese). No to Hattatsu (Tokyo). 2012;44(347).

[9] Takahashi R TMKJSSCSea Takayanagi M. EA 14year-old boy with encephalopathy with diverse symptoms and transient splenial lesion (in Japanese). The Journal of Sendai City Hospital (Sendai). 2012;32(537):115-9.

[10] Ka A, Britton P, Troedson C, Webster R, Procopis P, Ging J, et al. Mild encephalopathy with reversible splenial lesion: An important differential of encephalitis. European Journal of Paediatric Neurology. 2015 May;19(3):377-382. Publ(Med.

[11] Chen WX, Liu HS, Yang SD, Zeng SH, Gao YY, Du ZH, et al. Reversible splenial lesion syndrome in children: Retrospective study and summary of case series. Brain and Development. 2016 Nov;38(10):915-927. PublMed.
[12] Kometani H, Kawatani M, Ohta G, Okazaki S, Ogura K, Yasutomi M, et al. Marked elevation of interleukin- 6 in mild encephalopathy with a reversible splenial lesion (MERS) associated with acute focal bacterial nephritis caused by Enterococcus faecalis. Brain and Development. 2014 Jun;36(6):551-553. PublMed. 